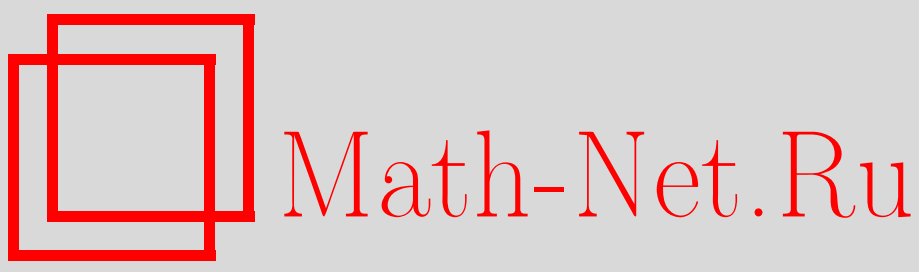

П. В. Тумаркин, Подсистемы корней полного ранга в гиперболических системах корней, Матем. сб., 2004, том 195, номер 1, 129-142

DOI: https://doi.org/10.4213/sm796

Использование Общероссийского математического портала Math-Net.Ru подразумевает, что вы прочитали и согласны с пользовательским соглашением

http://www . mathnet.ru/rus/agreement

Параметры загрузки:

IP: 34.239 .49 .27

26 апреля 2023 г., $12: 33: 58$ 
УДК 512.817 .72

\author{
П.В. Тумаркин
}

\title{
Подсистемы корней полного ранга в гиперболических системах корней
}

\begin{abstract}
Алгебра Каца-Муди назьвается гиперболической, если она построена по обобщенной матрице Картана гиперболического типа. Мы исследуем подсистемы корней в системах корней алгебр такого вида. Основнњм резултатом настоящей работы является классификация регулярных гиперболических подалгебр полного ранга гиперболических алгебр Каца-Муди.
\end{abstract}

Библиография: 9 названий.

\section{Введение}

Симметризуемая обобшенная матрица Картана называется матрицей гиперболического типа, если при симметризации получается матрица сигнатуры $(n, 1)$, любая главная подматрица которой имеет конечный или аффинньй тип. Пусть $A-$ обобщенная матрица Картана гиперболического типа. Следуя [1], по матрице $A$ можно построить алгебру Каца-Муди $\mathfrak{g}(A)$. Согласно [2] группа Вейля ее системы корней $\Delta(A)$ является группой Кокстера. Фундаментальной камерой группы Вейля является $n$-мерньй гиперболический симплекс Кокстера конечного объема (рассмотренный в линейной модели гиперболического пространства), не имеющий двугранных углов отличных от $\pi / 2, \pi / 3, \pi / 4$ и $\pi / 6$. При $n=2$ угол может также равняться нулю.

По аналогии с [3] назовем подалгебру $\mathfrak{g}_{1} \subset \mathfrak{g}(A)$ регулярной, если она инвариантна относительно некоторой картановской подалгебры $\mathfrak{h}$ алгебры $\mathfrak{g}(A)$. Иначе говоря, $\mathfrak{g}_{1} \subset \mathfrak{g}(A)$ регулярна, если для нее можно построить базис из элементов $\mathfrak{h}$ и корневых векторов алгебры $\mathfrak{g}(A)$ относительно $\mathfrak{h}$. Нас будут интересовать те регулярные подалгебры полного ранга, которые могут быть построены как алгебры Каца-Муди $\mathfrak{g}_{1}\left(A_{1}\right)$ по некоторой обобщенной матрице Картана гиперболического типа $A_{1}$.

Каждой такой подалгебре алгебры $\mathfrak{g}(A)$ соответствует система корней $\Delta_{1}\left(A_{1}\right) \subset$ $\Delta(A)$, удовлетворяющая условию:

$$
\text { если } \alpha, \beta \in \Delta_{1} \text { и } \alpha+\beta \in \Delta \text {, то } \alpha+\beta \in \Delta_{1} \text {. }
$$

И обратно: имея систему корней, лежащую в гиперболической системе корней и удовлетворяющую условию (*), мы можем построить подалгебру указанного типа в алгебре $\mathfrak{g}(A)$.

Гиперболической системой корней мы будем называть систему корней алгебры Каца-Муди, построенной по обобщенной матрице Картана гиперболического 
типа. Рангом системы корней назовем ранг соответствующей обобщенной матрицы Картана. Фундаментальная камера группы Вейля гиперболической системы корней ранга $k$ является гиперболическим симплексом размерности $k-1$.

Пусть $\Delta$ - система корней. Подсистемой корней в $\Delta$ называется система корней $\Delta_{1} \subset \Delta$, для которой выполнено условие $(*)$.

Классификация подсистем корней в эллиптических системах корней получена Дынкиным в [3].

Настоящая работа посвящена классификации гиперболических подсистем корней полного ранга в гиперболических системах корней.

Рассмотрим гиперболическую подсистему корней полного ранга $\Delta_{1}$ в гиперболической системе корней $\Delta$. Фундаментальной камерой группы Вейля $W_{1}$ системы корней $\Delta_{1}$ является некоторый симплекс Кокстера $F_{1}$, а фундаментальной камерой группы Вейля $W$ системы корней $\Delta$ - некоторый симплекс Кокстера $F$. Группа $W_{1}$ порождена отражениями относительно граней симплекса $F_{1}$, а $W$ - отражениями относительно граней симплекса $F$. Поскольку $W_{1}$ является подгруппой группы $W$, симплекс $F_{1}$ состоит из нескольких копий симплекса $F$, причем если две копии имеют обшую гран, то они симметричны относительно нее. Такое разбиение $\left(F, F_{1}\right)$ симплекса $F_{1}$ на симплексы $F$ мы будем называть минимальным, если $W_{1}$ является максимальной (по включению) собственной подгруппой в $W$, порожденной отражениями. Все минимальные разбиения гиперболических симплексов Кокстера конечного объема перечислены в [4]-[6].

В дальнейшем, говоря о симплексах, мы подразумеваем гиперболический симплекс Кокстера конечного объема, не имеющий двугранных углов отличных от $\pi / 2, \pi / 3, \pi / 4$ и $\pi / 6$ (при $n=2$ допускаются также углы равные нулю).

В $\S 1$ (теорема 1) будет доказано, что любому минимальному разбиению гиперболических симплексов соответствует некоторая подсистема корней в гиперболической системе корней. В $\S 2$ (теорема 2 ) будет доказано, что любому разбиению гиперболического симплекса на гиперболические симплексы соответствует некоторая подсистема корней. Классификация подсистем корней приведена на рис. $1-19$.

Автор выражает благодарность Э. Б. Винбергу за внимание к работе и полезные замечания.

\section{§1. Максимальные подгруппы}

В дальнейшем мы будем пользоваться следуюшими обозначениями: $A=\left(a_{i j}\right)$ симметризуемая обобщенная матрица Картана гиперболического типа; $\Delta-$ соответствующая система корней; $\alpha_{1}, \ldots, \alpha_{n+1}$ - простые корни; $L=\sum_{i=1}^{n+1} \mathbb{Z} \alpha_{i}$-соответствуюшая решетка корней. Группа Вейля $W_{F}$ системы корней $\Delta$ порождена отражениями в гранях фундаментальной камеры - симплекса $F$. Грани $F$ - гиперплоскости, на которых обрашаются в нуль простые корни. Далее, $\Delta^{\vee}$ и $L^{\vee}-$ система и решетка корней, соответствуюшие обобшенной матрице Картана $A^{t}$ (ей соответствует система простых корней с тем же фундаментальным симплексом $F$, но с другими длинами); $\Delta_{1} \subset \Delta$ - гиперболическая система корней, решетка корней которой, $L_{1}$, является подрешеткой полного ранга в $L ; F_{1}$ - фундаментальный симплекс для группы Вейля $W_{F_{1}}$ системы корней $\Delta_{1}$.

Для изображения систем корней мы будем использовать схемы Дьнкина. Каж- 
дая вершина $v_{i}$ схемы Дынкина системы $\Delta$ соответствует простому корню $\alpha_{i}$. Вершины $v_{i}$ и $v_{j}$ соединены ребром кратности $k=a_{i j} a_{j i}$, если $k=1,2$ или 3 . Если $k=0$, то вершины не соединены, а если $k=4$ (это возможно только при $n=2$ ), то вершины соединены толстым ребром. Если $\left|a_{i j}\right|<\left|a_{j i}\right|$, то соответствуюшее ребро ориентируется стрелкой, направленной от $v_{i}$ к $v_{j}$.

Мы воспользуемся следующим описанием системы корней гиперболической алгебры (см. [1; предложение 5.10]). Гиперболическая система корней $\Delta$ состоит из двух частей: множество вешественных корней $\Delta^{\text {re }}$ и множество мнимых корней $\Delta^{\mathrm{im}}$, где

$$
\Delta^{\mathrm{re}}=W\left(\alpha_{1}\right) \cup \cdots \cup W\left(\alpha_{n+1}\right), \quad \Delta^{\mathrm{im}}=\{\alpha \in L \mid(\alpha \mid \alpha) \leqslant 0\} .
$$

Лемма 1. Пусть $\left(F, F_{1}\right)$ - минимальное разбиение. Тогда следующие условия эквивалентнь:

1) $L_{1}-$ собственная подрешетка в $L$;

2) $\Delta_{1}=\Delta \cap L_{1}$;

3) $\Delta_{1}$ является подсистемой корней в $\Delta$;

4) условие $(*)$ выполняется для $\Delta_{1}^{\mathrm{re}} \subset \Delta^{\mathrm{re}}$.

ДоказАТЕЛьство. 1) $\rightarrow 2$ ). Для $\Delta_{1}^{\mathrm{im}}$ утверждение очевидно.

Пусть найдется $\alpha \in \Delta^{\text {re }}$ такой, что $\alpha \in L$, но $\alpha \notin \Delta_{1}$. Рассмотрим подгруппу $G$ группы $W_{F}$, порожденную отражениями относительно всех корней, лежащих в $L_{1}$. Очевидно, $W_{F_{1}} \subset G$. Поскольку $W_{F_{1}}$ максимальна в $W_{F}$ и $G \neq W_{F_{1}}$, мы получаем, что $G=W_{F}$. Тогда все простые корни системы $\Delta$ представляются как линейные комбинации корней из $L_{1}$ с целыми коэффициентами, а следовательно, и сами лежат в $L_{1}$. То есть $L=L_{1}$.

$2) \rightarrow 3$ ). Пусть $\alpha, \beta \in \Delta_{1}$. Тогда $\alpha, \beta, \alpha+\beta \in L_{1}$. Если $\alpha+\beta \in \Delta$, то $\alpha+\beta \in \Delta \cap L_{1}$, т.е. $\alpha+\beta \in \Delta_{1}$.

$3) \rightarrow 4)$. Доказательство очевидно.

$4) \rightarrow 1)$. Пусть $L$ совпадает с $L_{1}$. Предположим, что у $F_{1}$ есть разрезанньй двугранный угол, т.е. зеркало отражения из группы $W_{F}$ разрезает угол симплекса $F_{1}$. Пусть $\alpha, \beta \in \Delta_{1}^{\text {re }}$ - корни, обрашаюшиеся в нуль на гранях этого угла (мы считаем, что корни - внешние нормали к граням угла). Тогда $\alpha-\beta \in L_{1}$ обрашается в нуль на одном из зеркал, разрезающих угол. Следовательно, $c(\alpha-\beta) \in \Delta^{\text {re }}$ при некотором $c>0$. По предположению решетка $L$ совпадает с $L_{1}$, поэтому $c(\alpha-\beta) \in L_{1}$. Мы можем считать, что $\alpha$ и $\beta$ - простые корни в $\Delta_{1}$. Тогда $\alpha-\beta \notin \Delta_{1}^{\text {re }}$. Решетка $L_{1}$ натянута на простые корни системы $\Delta_{1}$, а следовательно, $c \in \mathbb{Z}$. Поскольку для $\Delta_{1}^{\mathrm{re}} \subset \Delta^{\mathrm{re}}$ вьполнено условие $(*)$ и $\alpha-\beta \notin \Delta_{1}^{\text {re }}$, мы получаем, что $c \neq 1$. Учитывая, что $(\alpha \mid \beta) \leqslant 0$, при $c \geqslant 2$ корень $c(\alpha-\beta)$ оказывается более чем в два раза длиннее $\alpha$, что невозможно, так как они не ортогональны друг другу.

Пусть теперь у $F_{1}$ нет разрезанного угла. Тогда пара $\left(F, F_{1}\right)$ - это одна из шести пар, приведенных в таблице 1 (см. [6]). Поскольку ни один из этих симплексов не имеет двугранных углов отличных от $\pi / 2$ и $\pi / 3$, каждому из них соответствует ровно одна система корней. Непосредственная проверка показывает, что в каждом из этих случаев корни подсистемы порождают подрешетку индекса два в решетке корней. 


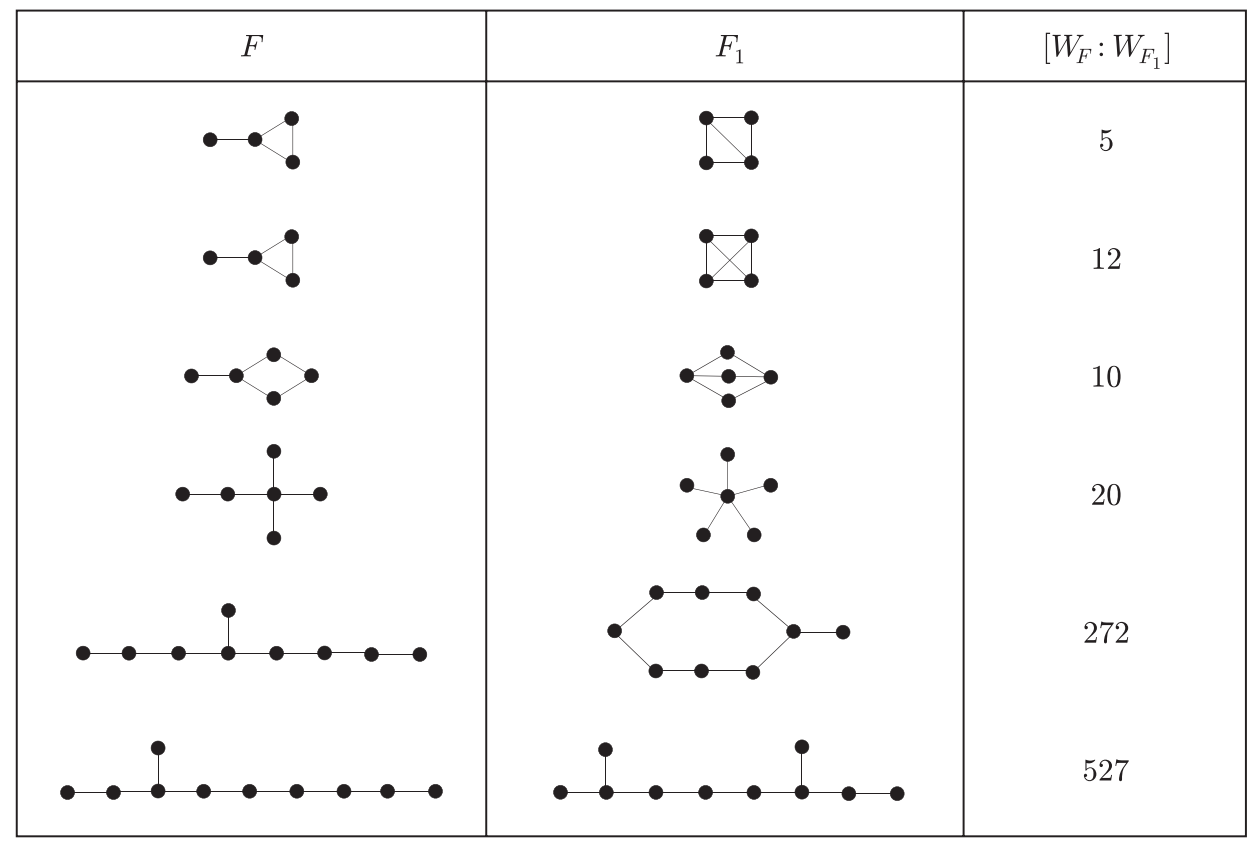

ТАБлицА 1. Минимальные разбиения с неразрезанньми двугранньми углами

ЗАмечАниЕ. Минимальность разбиения не используется при доказательстве второй импликации. Это понадобится нам при исследовании неминимальных разбиений.

Одному и тому же симплексу $F$ может соответствовать несколько систем корней. Действительно, если у $F$ есть хотя бы один угол отличный от $\pi / 2$ и $\pi / 3$, то мы можем разньми способами задать длины корней (см. $\S 3)$. Далее будет доказано, что для любого минимального разбиения $\left(F, F_{1}\right)$ можно выбрать такую систему корней $\Delta$ (с фундаментальньм симплексом $F$ ), что корни, определяющие симплекс $F_{1}$, порождают собственную подрешетку в $L$. По лемме 1 для системы, соответствуюшей симплексу $F_{1}$, будет выполняться условие $(*)$.

Рассмотрим сначала случай, когда симплекс $F_{1}$ состоит из двух экземпляров симплекса $F$.

Лемма 2. Пусть $\left[W_{F}: W_{F_{1}}\right]=2$. Рассмотрим любую систему корней $\Delta$ с фундаментальным симплексом $F$. Тогда корни либо из $\Delta$, либо из $\Delta^{\vee}$, обращающиеся в нуль на гранях $F_{1}$, порождают собственную подрешетку $L_{1}$ либо в $L$, либо в $L^{\vee}$ соответственно. Индекс подрешетки равен двум, трем или четьрем.

ДоказАтЕльство. Симплекс $F_{1}$ является объединением симплекса $F$ и его образа, полученного отражением относительно одной из граней. Можно считать, что $\alpha_{1}$ - корень, определяющий эту грань. Поскольку у $F_{1}$ и $F$ все грани, кроме одной, общие, только один из корней $\alpha_{2}, \ldots, \alpha_{n+1}$ не ортогонален $\alpha_{1}$.

Пусть $\alpha_{2}$ не ортогонален $\alpha_{1}$. Можно считать, что $\left|a_{21}\right| \leqslant\left|a_{12}\right|$, иначе вместо матрицы $A$ рассмотрим матрицу $A^{t}$. Тогда многогранник $F_{1}$ ограничен гранями, соответствующими корням $\alpha_{2}-a_{12} \alpha_{1}, \alpha_{2}, \alpha_{3}, \ldots, \alpha_{n+1}$. 
Поскольку $A$ - симметризуемая матрица гиперболического типа, то $a_{12}=-1$, $-2,-3$ или $-4\left(-4\right.$ может встречаться только в том случае, когда $F_{1}$ и $F-$ неограниченные треугольники). Если $a_{12}=-1$, то угол между гранями, соответствующими $\alpha_{2}$ и $\alpha_{2}-a_{12} \alpha_{1}$, равен $2 \pi / 3$ и группа $W_{F_{1}}$ совпадает с $W_{F}$.

Поэтому либо $a_{12}=-2$, либо $a_{12}=-3$, либо $a_{12}=-4$. Следовательно, $L_{1}=\mathbb{Z}\left(\alpha_{2}-a_{12} \alpha_{1}\right)+\mathbb{Z} \alpha_{2}+\mathbb{Z} \alpha_{3}+\cdots+\mathbb{Z} \alpha_{n+1}$ является подрешеткой индекса $\left|a_{12}\right|$ в решетке $L=\sum_{i=1}^{n+1} \mathbb{Z} \alpha_{i}$.

Перейдем теперь к обшему случаю.

Tеорема 1. Пусть $W_{F_{1}}-$ максимальная подгруппа группь $W_{F}$. Рассмотрим любую систему корней $\Delta$ с фундаментальным симплексом $F$. Тогда корни либо из $\Delta$, либо из $\Delta^{\vee}$, обращающиеся в нуль на гранях $F_{1}$, порождают собственную подрешетку $L_{1}$ либо в $L$, либо в $L^{\vee}$ соответственно.

ДоКАЗАТЕЛЬСТво. Поскольку $W_{F_{1}}$ является подгруппой группы $W_{F}$, симплекс $F_{1}$ состоит из нескольких копий симплекса $F$, причем если две копии имеют общую грань, то они симметричны относительно нее.

Рассмотрев все минимальные разбиения (они описаны в работах [4]-[6]), можно заметить, что для любого минимального разбиения $\left(F, F_{1}\right)$ у симплекса $F_{1}$ всегда есть хотя бы одна вершина, стабилизатор которой в $W_{F}$ совпадает с ее стабилизатором в $W_{F_{1}}$. Другими словами, можно считать, что у симплексов $F$ и $F_{1}$ есть $n$ обших граней - в точности те грани, которые в пересечении дают эту вершину.

Пусть $\alpha_{1}, \ldots, \alpha_{n}$ - корни, обрашаюшиеся в нуль на обших для $F$ и $F_{1}$ гранях, а $\alpha_{n+1}$ и $\alpha_{F_{1}}-$ корни, соответствующие оставшимся граням симплексов $F$ и $F_{1}$ соответственно. Выразим $\alpha_{F_{1}}$ через $\alpha_{1}, \ldots, \alpha_{n+1}$. Тогда индекс подрешетки, порожденной векторами $\alpha_{1}, \ldots, \alpha_{n}, \alpha_{F_{1}}$, равен коэффициенту при $\alpha_{n+1}$.

Теперь возьмем минимальное разбиение $\left(F, F_{1}\right)$ и систему корней $\Delta$ с фундаментальным симплексом $F$ и подсчитаем коэффициент при $\alpha_{n+1}$ в разложении $\alpha_{F_{1}}$. Если он отличен от единишы, то мы получаем собственную подрешетку (коэффициент не может быть отрицательным, поскольку $\alpha_{F_{1}}-$ положительный корень; он также не может равняться нулю, так как тогда простые корни подсистемы были бы линейно зависимы). Пусть он равен единице. Поменяем направление всех стрелок в схеме Дынкина системы корней $\Delta$. Другими словами, рассмотрим систему корней $\Delta^{\vee}$, отличающуюся от $\Delta$ только длинами корней, причем самые короткие корни станут самыми длинными и наоборот. Непосредственньм вычислением можно проверить, что в этой системе коэффициент при $\alpha_{n+1}$ в разложении $\alpha_{F_{1}}$ отличен от единицы. Почти во всех случаях коэффициент равен двум. Более точно, индекс подрешетки не равный двум встречается только в размерностях 2 и 3 (см. рис. $1-7$ ).

\section{§2. Немаксимальные подгруппы}

В этом параграфе для каждого неминимального разбиения $\left(F, F_{1}\right)$ мы найдем подсистему корней $\Delta_{1}$ (с фундаментальньм симплексом $F_{1}$ соответствующей группы Вейля) в системе корней $\Delta$ (с фундаментальньм симплексом $F$ ). Вообше говоря, для некоторых пар $\left(F, F_{1}\right)$ такую пару систем корней $\Delta_{1} \subset \Delta$ можно выбрать неединственным образом.

ЛЕмма 3. Пусть $\Delta_{1}-$ подсистема корней в $\Delta$, а $\Delta_{2}-$ подсистема корней в $\Delta_{1}$. Тогда $\Delta_{2} \subset \Delta$ также является подсистемой корней. 
ДокаЗАТЕЛЬство. Действительно, пусть $\alpha, \beta \in \Delta_{2}$ и $\alpha+\beta \in \Delta$. Поскольку $\Delta_{2} \subset \Delta_{1}$, имеем $\alpha, \beta \in \Delta_{1}$. Для $\Delta_{1} \subset \Delta$ условие (*) выполнено, а следовательно, $\alpha+\beta \in \Delta_{1}$. Но условие $(*)$ вьполнено и для $\Delta_{2} \subset \Delta_{1}$. Отсюда следует, что $\alpha+\beta \in \Delta_{2}$ и для $\Delta_{2} \subset \Delta$ вьполнено условие $(*)$.

Условия леммы, вообше говоря, не являются необходимыми (см. §3). Однако верно следуюшее утверждение:

ЛЕмма 4. Пусть $\Delta_{2} \subset \Delta_{1} \subset \Delta$, и пусть $\Delta_{2} \subset \Delta_{1}$ не является подсистемой корней. Тогда $\Delta_{2} \subset \Delta$ также не является подсистемой корней.

ДокАЗАТЕЛЬСтво. По условию леммы найдутся $\alpha, \beta \in \Delta_{2}$ и $\alpha+\beta \in \Delta_{1}$ такие, что $\alpha+\beta \notin \Delta_{2}$. Поскольку $\Delta_{1} \subset \Delta$, имеем $\alpha+\beta \in \Delta$. Отсюда следует, что для $\Delta_{2} \subset \Delta$ условие $(*)$ не вьполнено.

Из леммы 3 следует, что нам достаточно для каждого неминимального разбиения $\left(F, F_{1}\right)$ построить цепочку систем корней $\Delta_{k} \subset \Delta_{k-1} \subset \cdots \subset \Delta_{1} \subset \Delta$ так, что $\Delta_{k}$ соответствует симплексу $F_{1}, \Delta$ соответствует симплексу $F$ и для любого $i \leqslant k$ разбиение, соответствуюшее системе $\Delta_{i} \subset \Delta_{i-1}$, минимальное. Такую цепочку удается построить для всех неминимальных разбиений, кроме двух четырехмерных (см. рис. 10) и одного пятимерного (см. рис. 13). Подсистемы корней для этих трех разбиений приведены в $\S 3$.

Из сказанного выше мы получаем следуюшую теорему.

Теорема 2. Пусть F и $F_{1}-$-мерные гиперболические симплексы Кокстера конечного обвема, не имеющие двугранных углов отличных от $\pi / 2, \pi / 3$, $\pi / 4, \pi / 6$ и 0 . Пусть $W_{F_{1}}, W_{F}$ - группь, порожденные отражениями относительно гиперграней $F_{1}$ и $F$ соответственно, $u W_{F_{1}} \subset W_{F}$. Тогда найдутся система корней $\Delta$ с фундаментальным симплексом группь Вейля $F$ и система корней $\Delta_{1}$ с фундаментальным симплексом группы Вейля $F_{1}$ такие, что $\Delta_{1} \subset \Delta$ является подсистемой корней.

\section{§3. Классификация подсистем корней полного ранга}

Симплексы Кокстера в гиперболическом пространстве $\mathbb{H}^{n}$ сушествуют лишь при $n \leqslant 9$, причем число их конечно (см. [7]).

Симплексу Кокстера может соответствовать несколько систем корней. Все системы корней, соответствующие гиперболическому симплексу Кокстера конечного объема, получаются из соответствуюшей схемы Кокстера расстановкой стрелок на кратных и толстых ребрах (иначе говоря, указанием длин корней). Для того чтобы при расстановке стрелок получилась система корней, для каждого цикла в схеме Кокстера должно выполняться следуюшее условие: если в цикле нет толстых ребер, то в получившейся схеме Дынкина число ребер в цикле, направленных по часовой стрелке, должно быть равно числу ребер, направленных против часовой стрелки. Это правило должно вьполняться отдельно для двойных ребер и отдельно для тройных (заметим, что на схеме Дынкина системы простых корней угол $\pi / 6$ обозначается тройным ребром, в то время как на схеме Кокстера соответствуюшего симплекса - четверньм).

Если же в схеме есть цикл с толстьм ребром (а это возможно только при $n=2$ ), то условие выглядит немного по-другому. Если толстые ребра не направлены (т.е. 
соответствующие корни имеют одинаковую длину), то условие совпадает с написанным выше. А если есть направленное толстое ребро, то либо толстых направленных ребер два, они направлены в разные стороны и третий угол $-\pi / 2, \pi / 3$ или 0 , либо направленное толстое ребро одно и остальные два ребра двойные, направленные в противоположную сторону.

В обшем случае алгоритм отыскания всех систем корней с заданной группой Вейля описан в работе [8].

Для получения полной классификации систем корней мы делаем следуюшее. Рассмотрим минимальное разбиение $\left(F, F_{1}\right)$. Теперь расставим на схеме Кокстера симплекса $F$ стрелки всеми возможными способами и рассмотрим все системы корней, соответствуюшие симплексу $F_{1}$. Проделав эту процедуру для всех минимальных разбиений и рассмотрев также их композиции, мы получаем все гиперболические системы корней полного ранга в гиперболических системах корней.

Для получения классификации подсистем нам необходимо для каждой пары $\Delta_{1} \subset \Delta$ проверить, выполняется ли условие $(*)$. Для систем, соответствующих минимальньм разбиениям, это делается с помощью леммы 1: достаточно проверить, что система корней $\Delta_{1}$ порождает собственную подрешетку в решетке корней $L$. Как уже было отмечено выше, для неминимальных разбиений с помощью леммы 3 во многих случаях удается дать положительный ответ. Заметим, что в этом случае $\Delta_{1}=\Delta \cap L_{1}$, где $L_{1}$ - решетка корней системы $\Delta_{1}$. Лемма 4 также позволяет сушественно сократить вычисления для неминимальных разбиений: для большого количества пар $\Delta_{1} \subset \Delta$ удается сразу доказать, что условие $(*)$ не выполняется. В оставшихся случаях непосредственным вычислением проверяется, выполняется ли условие $(*)$. Оно выполнено для 18 пар систем корней. В каждом из этих восемнадцати случаев оказывается, что $\Delta_{1}=\Delta \cap L_{1}$. Вместе с леммой 1 и замечанием к ней это приводит нас к следующему результату.

ТЕОРема 3. Пусть $\Delta_{1} \subset \Delta$ - гиперболические системы корней одного ранга и $L_{1} \subset L$ - соответствующие решетки корней. Тогда следующие условия әквивалентни:

1) $\Delta_{1}=\Delta \cap L_{1}$

2) $\Delta_{1} \subset \Delta$ является подсистемой корней;

3) условие $(*)$ выполняется для $\Delta_{1}^{\mathrm{re}} \subset \Delta^{\mathrm{re}}$.

Ниже мы приводим полный список гиперболических подсистем корней полного ранга в гиперболических системах корней ранга 3-10.

На рис. 1-19 используются следующие обозначения. Если две схемы соединены, то система корней, соответствутствующая нижней схеме, содержится в системе корней, соответствующей верхней. На каждом ребре, соответствующем минимальному разбиению, написан индекс подгруппы. Если разбиение минимально, нижняя система является подсистемой верхней и индекс подрешетки не равен двум, то в скобках написан индекс подрешетки.

Виды соединений:

минимальное разбиение, условие (*) выполнено;

минимальное разбиение, но условие (*) не вьполнено;

неминимальное разбиение, условие $(*)$ вьполнено, но хотя 
бы для одного промежуточного минимального разбиения условие $(*)$ не выполняется.

Подсистема корней не соединена с системой корней в случае, если разбиение неминимальное и для всех промежуточных подсистем корней условие $(*)$ выполняется (лемма 3 ).

3.1. Подсистемы корней ранга 3. Существует всего три минимальных разбиения ограниченных треугольников Кокстера, не имеющих углов отличных от $\pi / 2, \pi / 3, \pi / 4$ и $\pi / 6$. Подсистемы корней, соответствующие ограниченньм треугольникам, описаны на рис. 1.

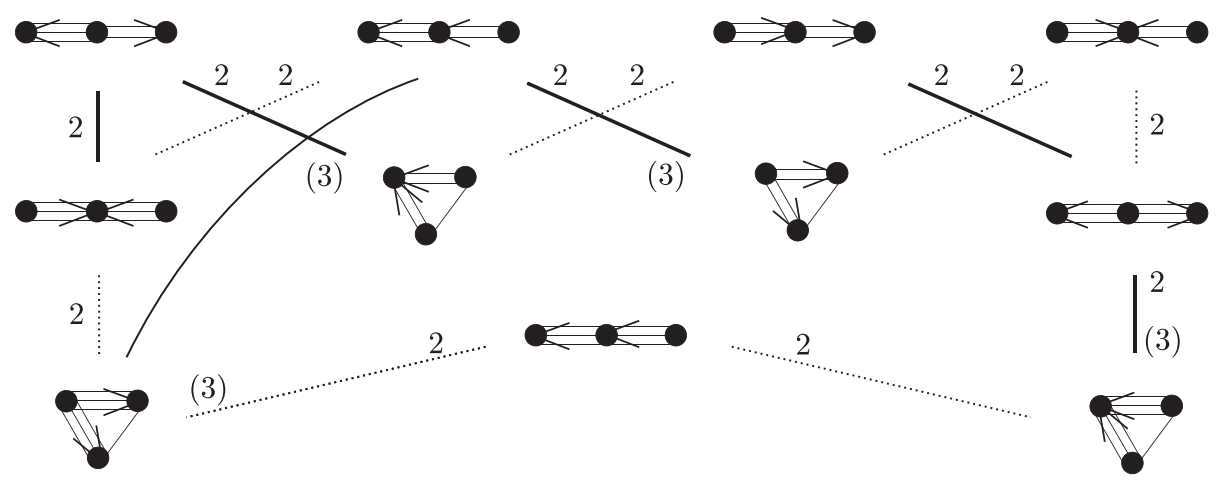

Рис. 1

Сушествует шесть классов соизмеримости неограниченных треугольников Кокстера, не имеющих углов отличных от $\pi / 2, \pi / 3, \pi / 4, \pi / 6$ и 0 . Пять из них содержат по одному элементу и, следовательно, не приводят к подсистемам корней. Оставшийся класс соизмеримости описан на рис. 2-4.

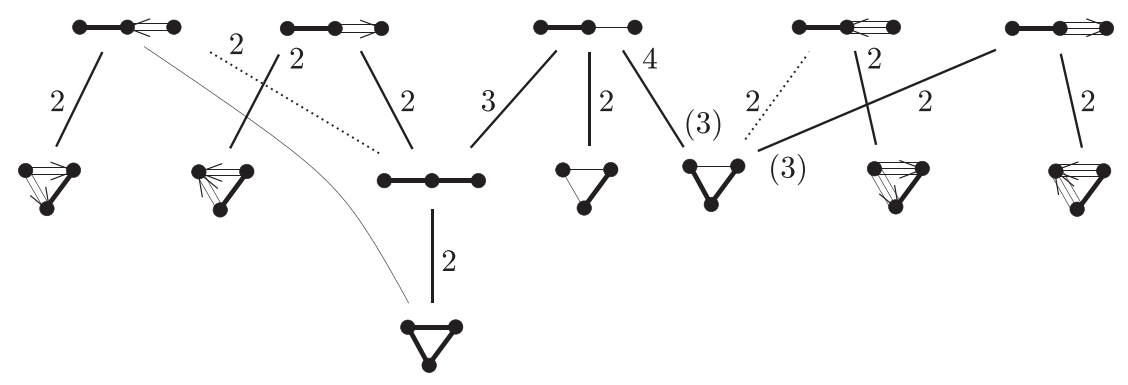

Рис. 2

На рис. 2 приведены подсистемы корней, схемы Дынкина которых не содержат ориентированных толстых ребер.

На рис. 3 и 4 описаны подсистемы корней, схемы Дьнкина которых содержат ориентированные толстые ребра.

3.2. Подсистемы корней ранга 4. Существует ровно одна система корней, фундаментальной камерой групшы Вейля которой является ограниченный тетраэдр Кокстера. 


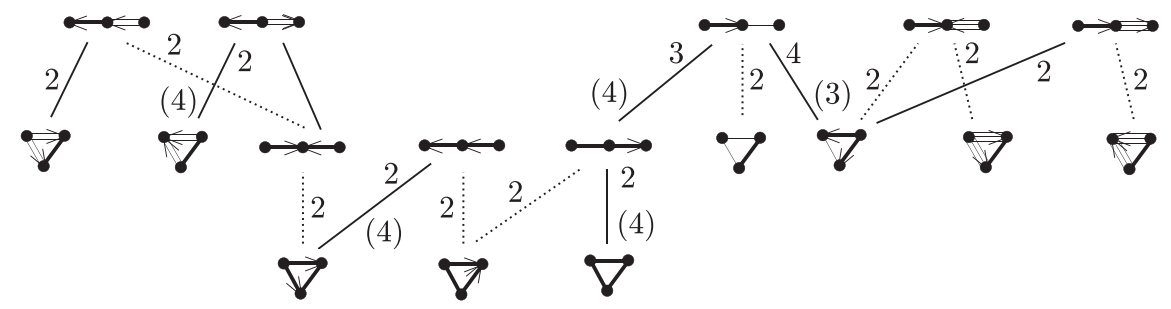

Рис. 3

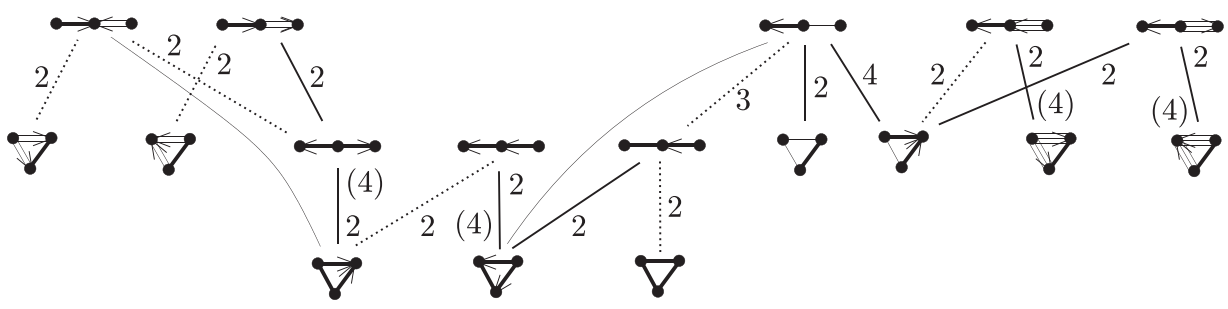

Рис. 4

Сушествует пять классов соизмеримости неограниченных тетраэдров Кокстеpa, не имеющих двугранных углов отличных от $\pi / 2, \pi / 3, \pi / 4$ и $\pi / 6$ (см. [9]). Три из них содержат по одному элементу и, следовательно, не приводят к подсистемам корней. Оставшиеся два класса описаны на рис. $5-7$ и на рис. $8-9$ соответственно.

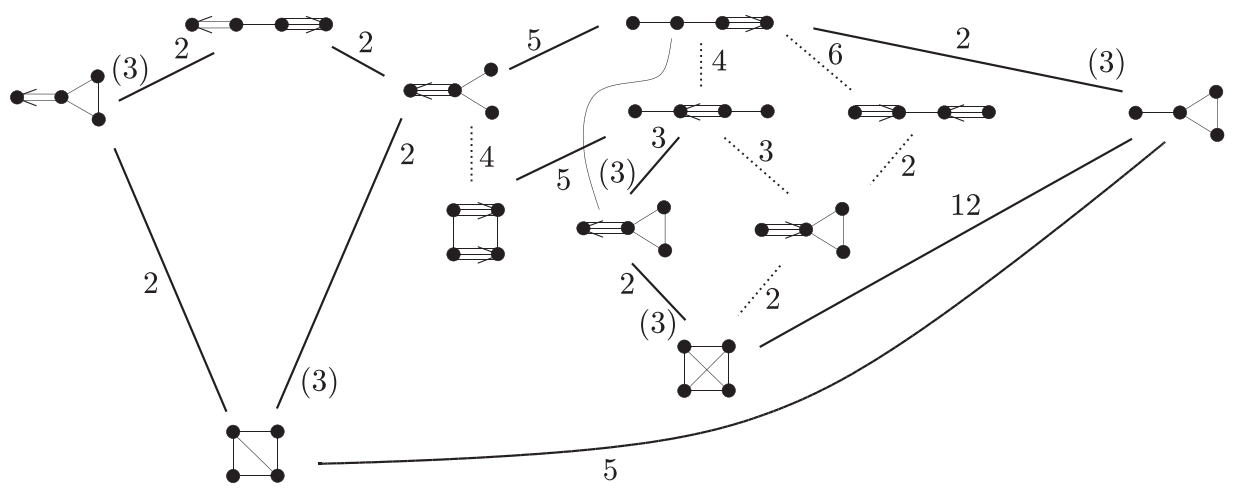

Рис. 5

Схемы на рис. 6 получены из схем рис. 5 заменой направления всех стрелок.

Все оставшиеся возможности расстановки стрелок приведены на рис. 7.

Системы корней, происходящие из второго класса соизмеримости, описаны на рис. 8 и 9.

На рис. 9 для наглядности изображения крайняя правая схема второго уровня сверху продублирована: она совпадает с крайней левой схемой того же уровня.

3.3. Подсистемы корней ранга 5. Согласно [9] (см. также [6]) существует ровно два класса соизмеримости четырехмерных гиперболических симлексов, не 
138

П.В. ТУМАРКИН

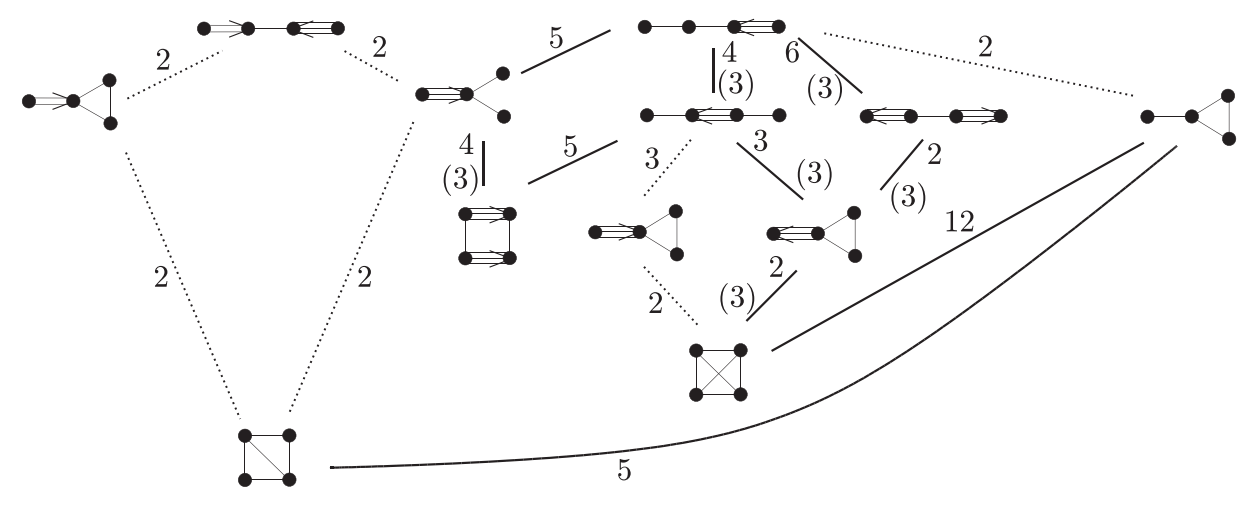

Рис. 6

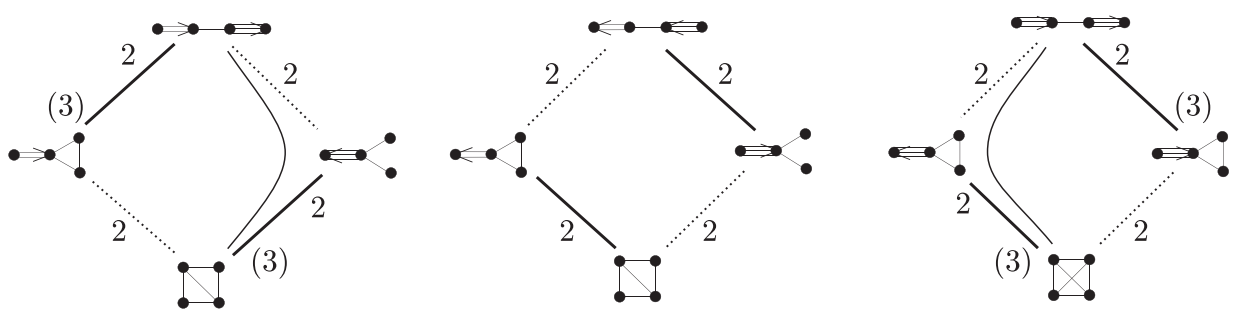

Рис. 7

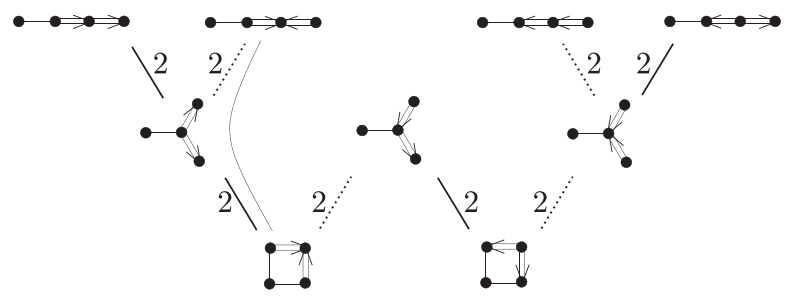

Рис. 8

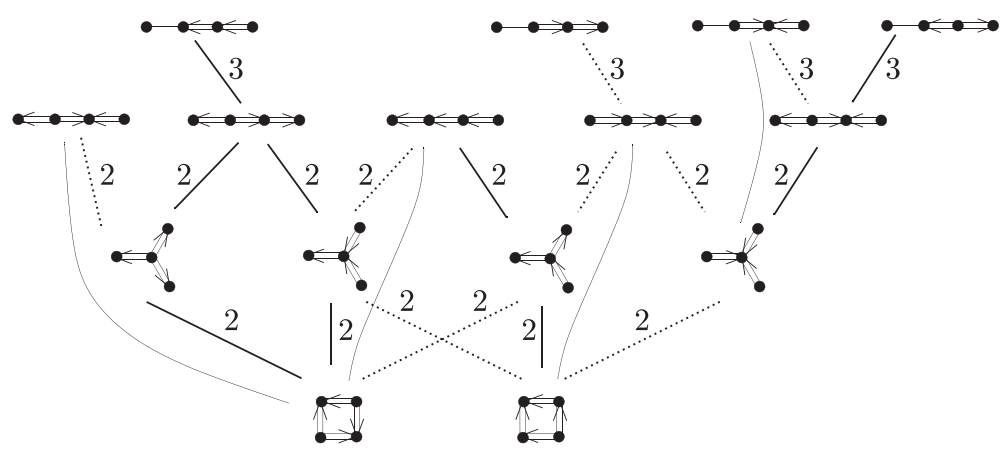

Рис. 9 
имеющих двугранных углов отличных от $\pi / 2, \pi / 3, \pi / 4$ и $\pi / 6$. Один из них содержит ровно один симплекс и не приводит к подсистемам корней. Оставшийся класс описан на рис. 10-12.

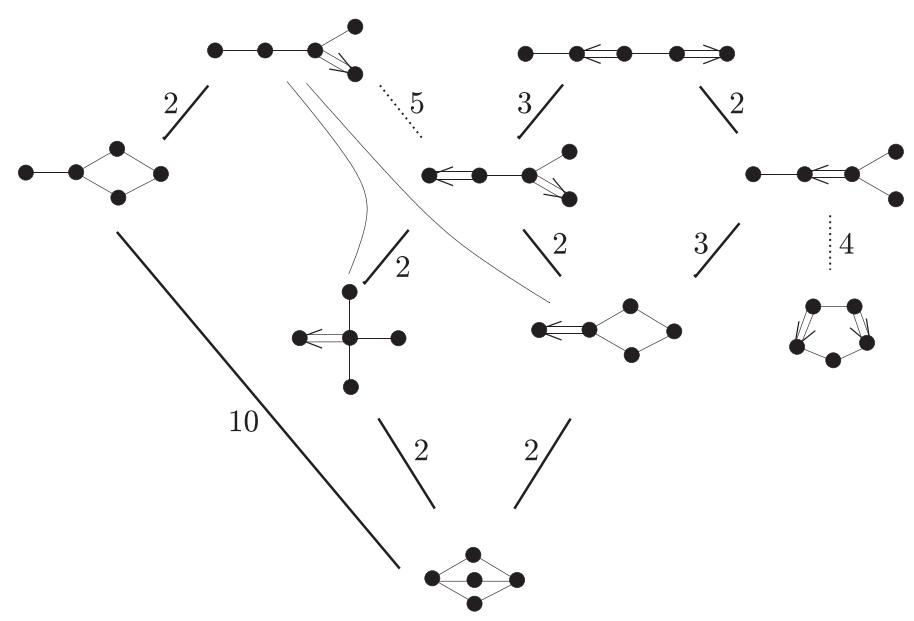

Рис. 10

Схемы на рис. 11 получены из схем рис. 10 заменой направления всех стрелок.

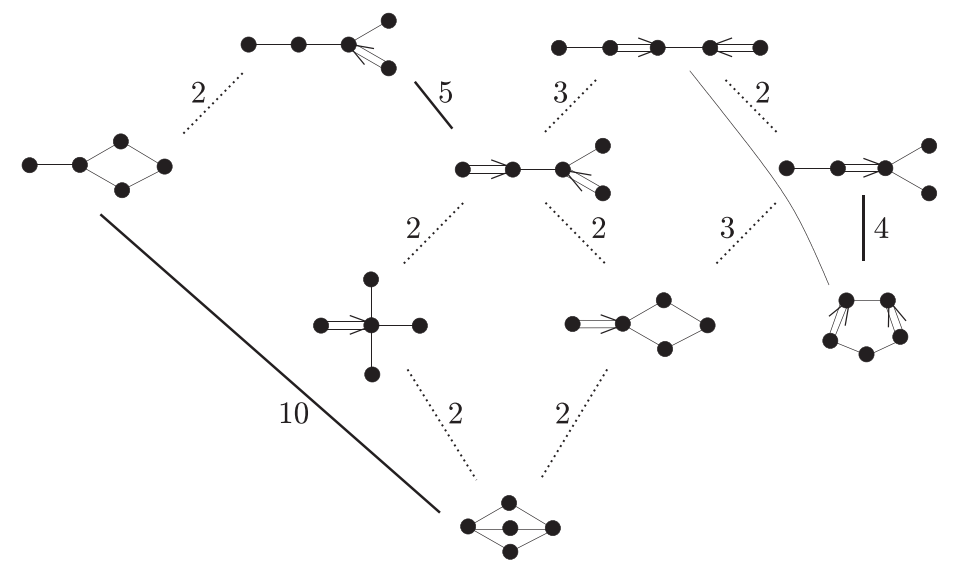

Рис. 11

Все оставшиеся возможности расстановки стрелок (все системы корней, содержащие вещественные корни трех различных длин) приведены на рис. 12 .

3.4. Подсистемы корней ранга 6. Согласно [9] (см. также [6]) сушествует ровно три класса соизмеримости пятимерных гиперболических симлексов. Два из них содержат по одному симплексу и не приводят к подсистемам корней. Оставшийся класс описан на рис. 13-15.

Схемы на рис. 14 получены из схем рис. 13 заменой направления всех стрелок. 

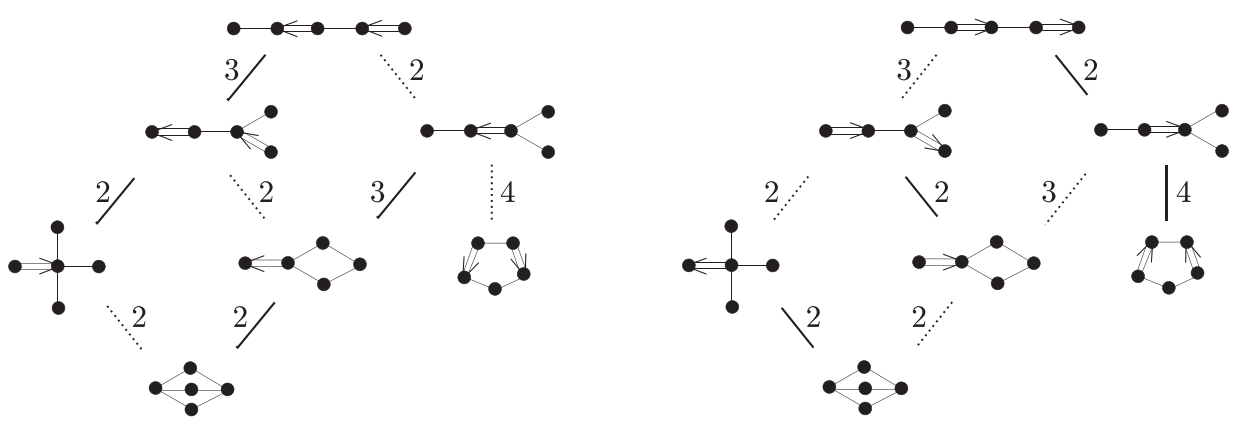

Рис. 12

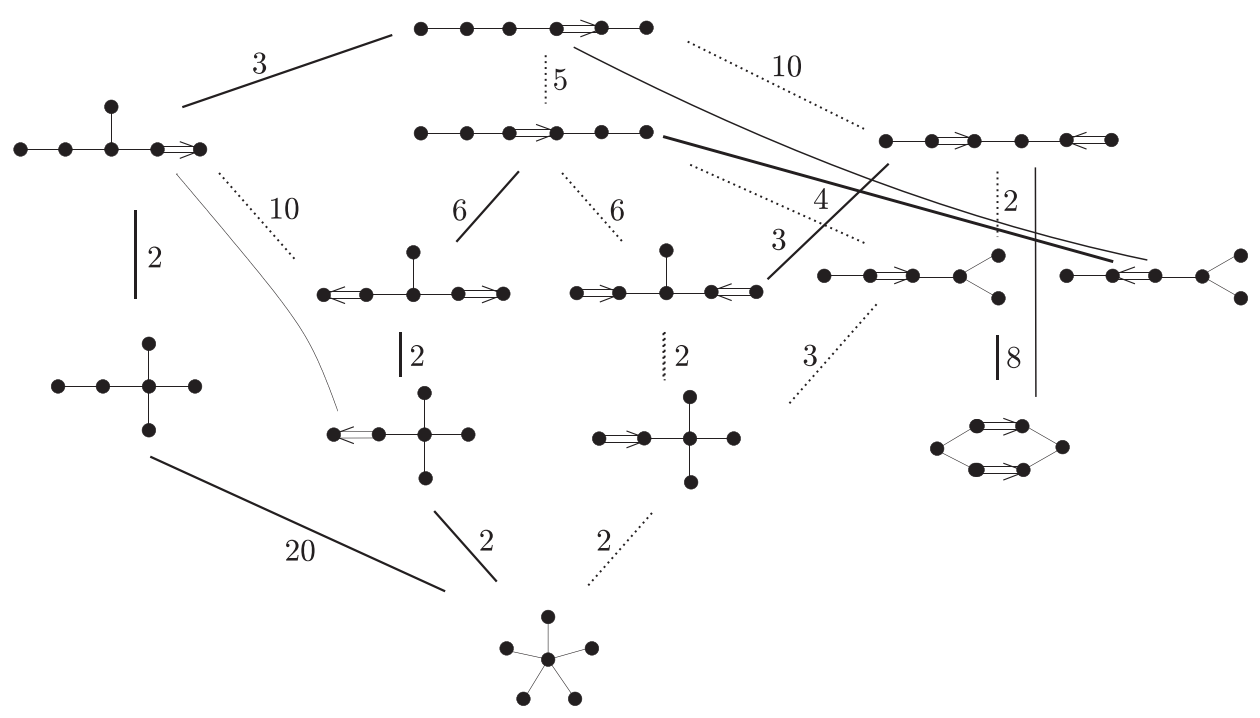

Рис. 13

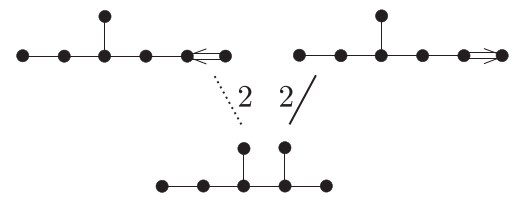

Рис. 16. Подсистемы корней ранга 7

Все оставшиеся возможности расстановки стрелок (все системы корней, содержащие вешественные корни трех различных длин) приведены на рис. 15 .

3.5. Подсистемы корней ранга 7-10. В размерностях 6-9 нет неминимальных разбиений симплексов. Все подсистемы корней ранга 7-10 приведены на рис. 16-19. 


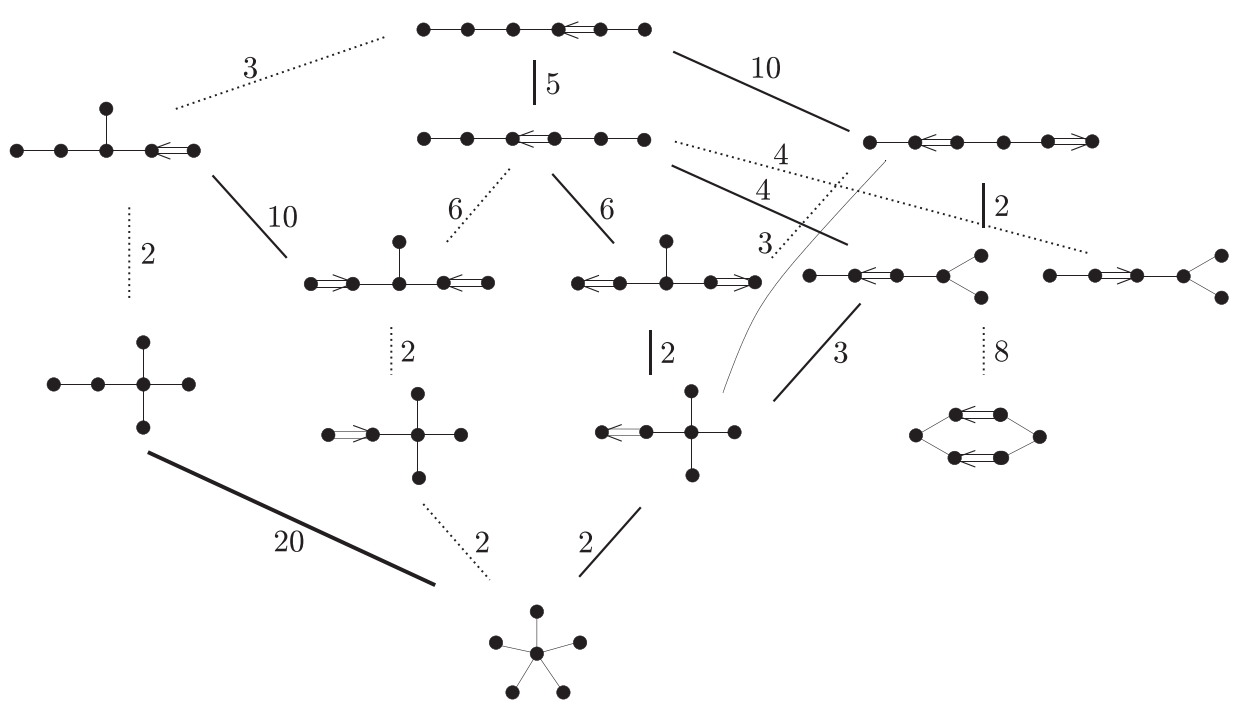

Рис. 14
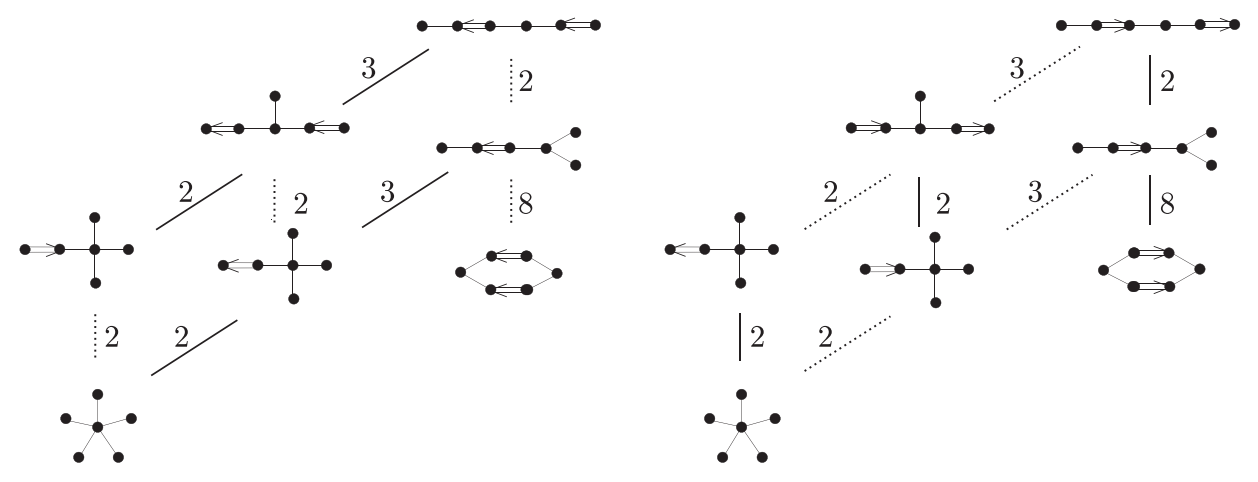

Рис. 15

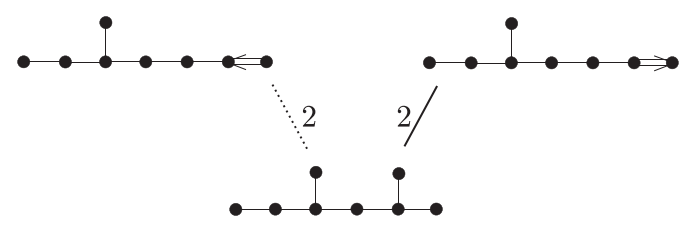

Рис. 17. Подсистемы корней ранга 8 

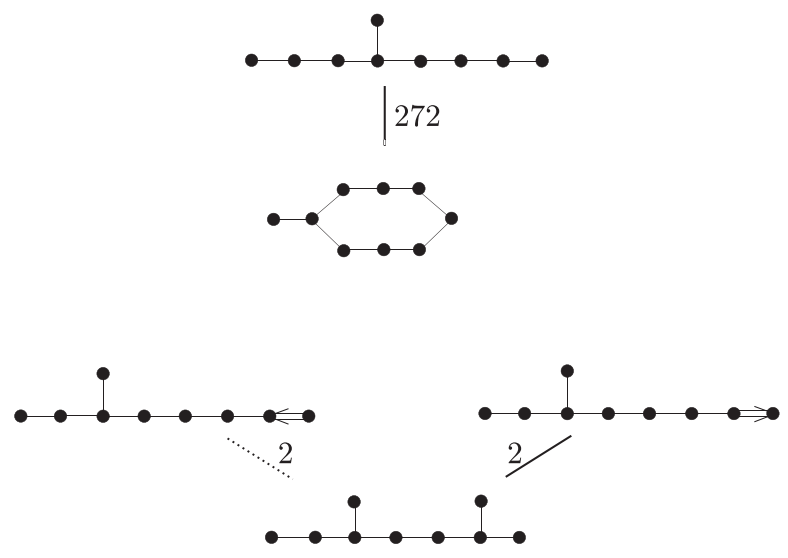

Рис. 18. Подсистемы корней ранга 9

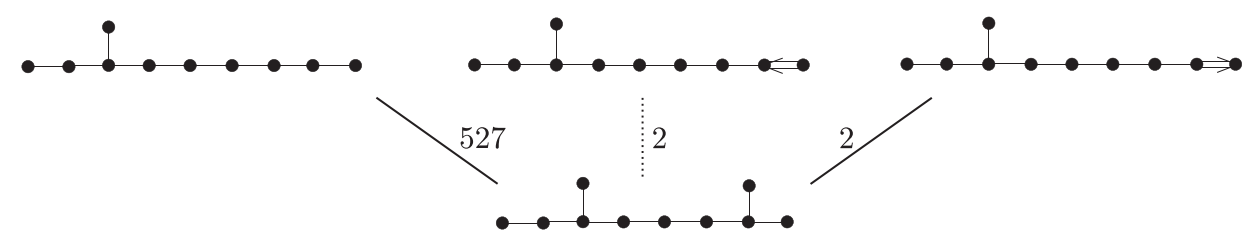

Рис. 19. Подсистемы корней ранга 10

\section{Список литературы}

1. Кач В. Бесконечномерные алгебры Ли. М.: Мир, 1993.

2. Винберг Э. Б. Дискретные линейные группы, порожденные отражениями // Изв. АН СССР. Сер. матем. 1971. Т. 35. С. 1072-1112.

3. Дынкин Е. Б. Полупростые подалгебры полупростых алгебр Ли // Матем. сб. 1952. T. 30 (72). № 2. C. 349-462.

4. Klimenko E., Sakuma $M$. Two-generator discrete subgroups of $\operatorname{Isom}\left(\mathbb{H}^{2}\right)$ containing orientation-reversing elements // Geom. Dedicata. 1998. V. 72. P. 247-282.

5. Felikson A. Coxeter decompositions of hyperbolic tetrahedra // Preprint. № 98-083. Bielefeld: Univ. Bielefeld, 1998.

6. Феликсон $A$. Кокстеровские разбиения гиперболических симплексов // Матем. сб. 2002. T. 193. № 12. C. 134-156.

7. Винберг Э. Б., Шварцман О. В. Дискретные группы движений пространств постоянной кривизны // Итоги науки и техники. Совр. проблемы матем. Фундамент. направления. Т. 29. М.: ВИНИТИ, 1988. С. 147-259.

8. Котова И. И. Описание систем корней с данной группой Вейля // Матем. заметки. 1998. T. 64. № 3. C. 397-402.

9. Johnson N. W., Kellerhals R., Ratcliffe J. G., Tschantz S. T. Commensurability classes of hyperbolic Coxeter groups // Linear Algebra Appl. 2002. V. 345. P. 119-147.

Независимый московский университет

Поступила в редакцию 29.11.2002 